\title{
Two types of non-homologous RNA recombination in brome mosaic virus
}

\author{
Magdalena Alejska, Nelli Malinowska, Anna Urbanowicz, Marek Figlerowicz ${ }^{\bowtie}$ \\ Institute of Bioorganic Chemistry, Polish Academy of Sciences, Poznań, Poland; ${ }^{\circledR}$-mail marekf@ibch.poznan.pl
}

Received: 01 December, 2004; revised: 07 April, 2005; accepted: 30 May, 2005

available on-line: 03 June, 2005

\begin{abstract}
Non-homologous RNA recombination is a process enabling the exchange of genetic material between various (related or unrelated) RNA-based viruses. Despite extensive investigations its molecular mechanism remains unclear. Studies on genetic recombination in brome mosaic virus (BMV) have shown that local hybridization between genomic RNAs induces frequent non-homologous crossovers. A detailed analysis of recombinant structures suggested that local complementary regions might be involved in two types of non-homologous recombination in BMV: site-specific and heteroduplex-mediated. To verify the above hypothesis and better recognize the mechanism of the phenomenon studied we have tested how the putative types of recombination are affected by a specific mutation in the BMV polymerase gene or by changes in RNA structure. The experiments undertaken revealed substantial differences between site-specific and heteroduplex-mediated recombination, indicating that they occur according to different mechanisms. The former can be classified as homology-assisted, and the latter as homology-independent. In addition to local RNA/RNA hybridization, short regions of homology are required for site-specific crossovers to occur. They are most efficiently mediated if one homologous sequence is located at the beginning of and the second just before a double-stranded region. At present it is difficult to state what is the mechanism of heteroduplex-mediated recombination. Earlier it was postulated that strong RNA/RNA interaction enforces template switching by the viral replicase. There are, however, several observations questioning this model and indicating that some other factors, which are still unknown, may influence heteroduplex-mediated crossovers.
\end{abstract}

Keywords: non-homologous RNA recombination, brome mosaic virus, heteroduplex-mediated RNA recombination, site-specific RNA recombination

Non-homologous RNA recombination is one of the major factors enabling the rapid evolution of RNA viruses (Eigen, 1996; Figlerowicz et al., 2003) and retroviruses (Pathak \& Hu, 1997). According to the definition, such recombination involves two different RNAs and generates products which differ markedly from the parental molecules (Lai, 1992). Because of the changes introduced into the viral genome, non-homologous recombinants rarely accumulate in vivo. However, they can sporadically acquire some new, advantageous features that allow them to spread and compete with their parental virus (Nagy \& Bujarski, 1992) or other already existing viruses (Khatchikian et al., 1989; Meyers et al., 1991). Despite extensive studies, the molecular mechanism of non-homologous recombination is still unclear. Most data suggest that recombinants are generated in compliance with the copy-choice model, i.e. as a result of template switching by the viral polymerase during the replication process. The polymerase begins RNA synthesis on one template, called the donor, and then switches to another template, called the acceptor (Nagy \& Simon, 1997; Figlerowicz \& Bujarski, 1998; Alejska et al., 2001).

In our studies on genetic RNA recombination we use a well-established in vivo system developed with BMV (Fig. 1) (Nagy \& Bujarski, 1993). BMV is a model positive-sense RNA virus of plants (Ahlquist, 1992). Its genome is composed of three RNA molecules called RNA1, RNA2 and RNA3. All three BMV RNAs possess an almost identical 3'-noncoding region (3'UTR) with a tRNA-like structure at the very end (Ahlquist, 1992). RNA1 and RNA2 encode BMV replicase proteins $1 \mathrm{a}$ and $2 \mathrm{a}$, respectively. The 1a protein contains two putative domains: the N-terminal helicase domain and the C-terminal domain,

Abbreviations: BMV, brome mosaic virus; RAS, recombinationally active sequence; UTR, untranslated region. 
involved in the capping of viral RNAs (Kroner et al., 1990). The 2a protein functions as an RNA-dependent RNA polymerase (Traynor et al., 1991). RNA3 is dispensable for BMV replication and encodes the movement and coat proteins (Ahlquist, 1992).

BMV was the first plant RNA virus for which genetic RNA recombination was demonstrated (Bujarski \& Kaesberg, 1986). Frequent recombination events were induced by inserting 40-140 nt complementary sequences into BMV genomic RNAs. Crossovers occurred within the local double-stranded region (RNA/RNA heteroduplex which recombining molecules were capable of forming) and therefore were classified as non-homologous (Nagy \& Bujarski, 1993; Figlerowicz, 2000). Based on a recently undertaken detailed analysis of recombinant structures we hypothesized that there are, in fact, two different types of non-homologous recombination in BMV: site-specific and heteroduplex-mediated (Figlerowicz, 2000) (Fig. 1D).

To verify the above hypothesis, improve our understanding of the mechanisms of the processes studied and optimize structural motifs supporting site-specific crossovers we have tested how both putative types of non-homologous recombination are affected by RNA structure or by a specific mutation in the BMV polymerase. Here we describe significant differences between site-specific and heteroduplexmediated recombination indicating distinct mechanisms for the two. We found that short homologous regions play a key role in site-specific recombination whereas heteroduplex-mediated recombination is homology-independent. Frequent site-specific crossovers are observed if one homologous sequence is located at the beginning and the second just before the local double-stranded structure on the donor RNA1 and acceptor RNA3, respectively. The data collected indicate that site-specific template switching by the BMV polymerase occurs preferentially during (-)RNA strand synthesis. Unfortunately, our studies did not provide any conclusive data concerning the mechanism of heteroduplex-mediated recombination.

\section{MATERIALS AND METHODS}

Materials. Plasmids pB1TP3, pB2TP5 and pB3TP7 are a generous gift of J.J. Bujarski (Northern Illinois University, DeKalb, USA). pB1TP3, pB2TP5 and pB3TP7 contain the full-length cDNA of BMV RNA1, RNA2 and RNA3, respectively. The construction of the pMag1-, pMag0- and pMag2-RNA3 plasmids containing cDNA of Mag1-, Mag0- and Mag2derivatives of BMV genomic RNA3 has already been described (Figlerowicz, 2000). Restriction enzymes (EcoRI, SpeI, XbaI), T7 RNA polymerase, RNasin, RQ DNase RNase free, MMLV-reverse transcriptase,
Taq polymerase and the pGEM cloning vector were from Promega. Nylon membrane HybondN and $\left[\alpha^{32} \mathrm{P}\right] \mathrm{CTP}$ were from Amersham

Deoxyoligonucleotides used for the construction of new BMV mutants (SpeI restriction site is underlined):

1. 5'-TCGAGGCCTCCTCGAGCAG-3',

2. 5'-TCGACTGCTCGAGGAGGCC-3',

3. 5'-ACTAGTACGCGTGGATCCTCGAGCA GAGGTCTCACACAG-3'

Primers used for specific RT-PCR amplification of the RNA3 3'-fragment (region where recombinant junction sites are located): first strand primer A, specific for all BMV RNAs 3'-end introducing EcoRI restriction site (underlined):

5'-CAGTGAATTCTGGTCTCTTTTAGAGATT-

TACAG-3' and second strand primer B representing the BMV RNA3 sequence between positions 1726 and 1751:

5'-CTGAAGCAGTGCCTGCTAAGGCGGTC-3' .

Plasmid construction. To prepare the $\mathrm{pIH}$ RNA1 plasmid, containing the cDNA of BMV IHRNA1, a short double-stranded DNA fragment (obtained by hybridization of deoxyoligonucleotides 1 and 2) was ligated into the XhoI linearized pB1TP3 plasmid. To construct the pHS-RNA3 plasmid containing the cDNA of HS-RNA3, the $76 \mathrm{nt}$ portion of pMag1-RNA3 (between the XbaI and SpeI restriction sites) was replaced with a $13 \mathrm{nt}$ longer dsDNA fragment obtained by PCR involving primers B and 3, and pMag1-RNA3 as template. The sequences of the newly prepared plasmids were confirmed by sequencing.

In vivo recombination assay. The previously described procedure was applied to test the recombination activity of the constructed BMV mutants (Nagy \& Bujarski, 1993). Briefly, infectious molecules of BMV RNA1 (wt or IH-RNA1), wtRNA2 and Mag0, Mag1-, Mag2- and HS-RNA3 derivatives were obtained by in vitro transcription for which EcoRI linearized plasmids pB1TP3, pIHRNA1, pB2TP5 and pMag0-, pMag1-, pMag2-, pHS-RNA3 were used. Four leaves of two Chenopodium quinoa plants (a local lesion host for BMV) were mechanically inoculated with mixtures containing BMV RNA1 (wt or IHRNA1), wtRNA2 and one of the RNA3 derivatives. After two weeks, when infection symptoms were well developed, the number of local lesions was counted to determine virus infectivity. Separate local lesions were excised and total RNA was isolated. The extracted RNA was subjected to RT-PCR involving primer A (first strand primer) and primer B (second strand primer), specific for amplification of the 3'-portion of RNA3 progenies (region where crossovers occur). The BMV-based recombination system was designed in such a way that the recombinants generated possessed a visibly shorter 3'-noncoding region than the parental Mag0-, Mag1-, Mag2- and 


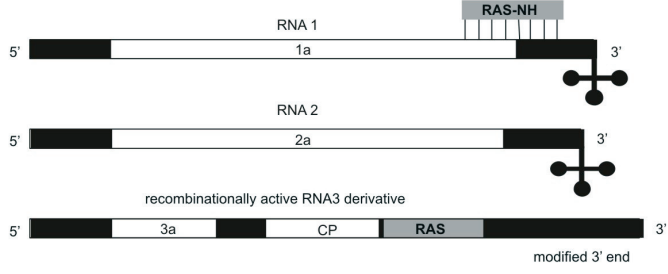

B.
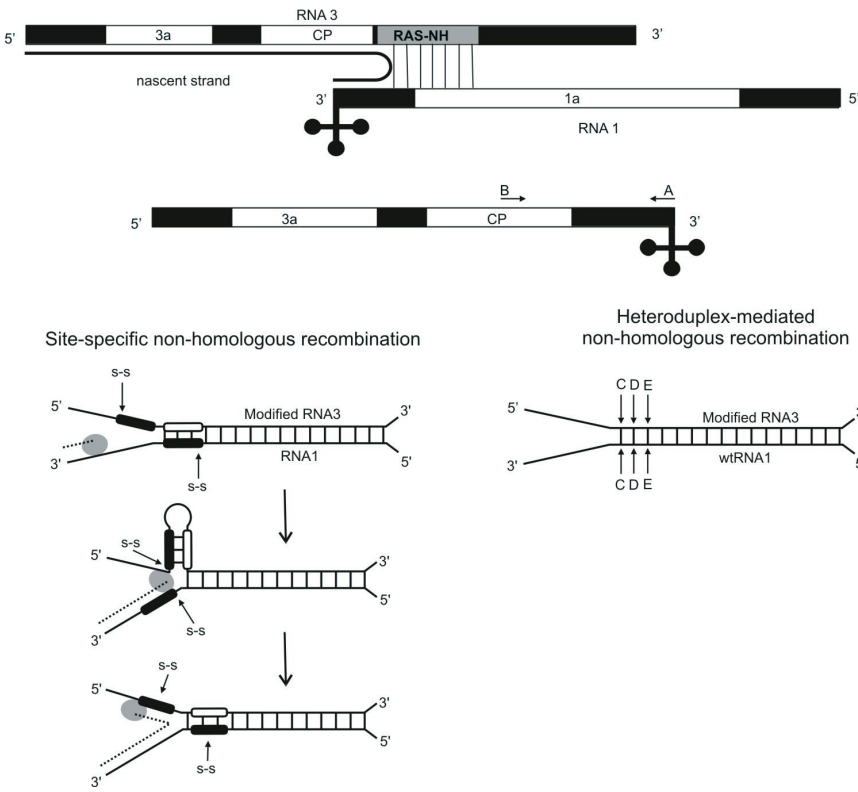

Heteroduplex-mediated non-homologous recombination

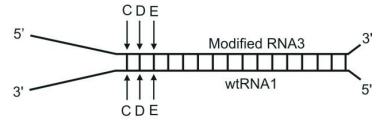

Figure 1. BMV in vivo non-homologous recombination system.

Particular fragments of the BMV genome are marked as follows: 3'UTRs, 5'UTRs and the intercistronic noncoding region are black, coding regions are white, the recombinationally active sequence (RAS) introduced into modified RNA3 is gray. Small arrows marked A and B represent the first and second strand primers used for the selective RT-PCR amplification of the RNA3 3'-portion - first strand primer A is complementary to the 3' end of all three BMV genomic RNAs (including the recombinationally active RNA3 derivative). Second strand primer B is specific for RNA3 amplification. A. Genome of the recombinationally active BMV mutant. The BMV genome is composed of three RNA molecules: RNA1, RNA2 and RNA3. RNA1 and RNA2 encode virus replicase proteins 1a and 2a, respectively. In RNA3, the movement protein (3a) and the coat protein (CP) are encoded. All three BMV genomic RNAs possess an almost identical 3'UTR adopting a tRNA-like structure. In our studies on genetic RNA recombination instead of a wild type (wt) virus we used a BMV mutant in which only the 3'UTR of RNA3 was modified (for details see Nagy \& Bujarski, 1993). The mutated virus possesses the entire genetic information necessary for BMV development, is stable in infected cells and starts to recombine if a RAS is inserted into the RNA3 derivative between the coding region and the modified 3'UTR. B. Non-homologous recombination. Frequent non-homologous crossovers were observed when an about 140 nt sequence complementary to the 3'-portion of RNA1 (sequence called RAS-NH) was introduced into the modified RNA3. Consequently, wtRNA1 and modified RNA3 were capable of forming a local double stranded structure (RNA1/RNA3 heteroduplex) that efficiently mediated recombination events. The proposed mechanism assumes that non-homologous recombinants are formed according to the copy-choice hypothesis. BMV polymerase cannot unwind the stable double-stranded region and switches from the RNA1 to the RNA3 template (Nagy \& Bujarski, 1993). C. BMV recombinant. Non-homologous recombination repairs the mutated virus. The highly modified $3^{\prime}$-end of RNA3 is replaced by the 3'UTR coming from RNA1 and conserved in all BMV RNAs. The recombinants replicate and accumulate better than parental RNA3 molecules so that the latter are out-competed from the infected cells. D. Putative RNA structural elements supporting sitespecific and heteroduplex-mediated non-homologous recombination in BMV. Complementary fragments of RNA1 and RNA3 are represented by solid lines. Black boxes symbolize two short homologous sequences while the region complementary to them is shown as a white box. The gray oval and short vertical lines symbolize BMV polymerase and local hybridization between recombining molecules, respectively. The location of recombinant junction sites is indicated by arrows and letters: s-s - site-specific recombinants; C, D, E - heteroduplex-mediated recombinants. Site-specific nonhomologous recombination is observed when the RNA1/RNA3 heteroduplex is accompanied by short homologous sequences specifically positioned in donor and acceptor templates. As a result the local double-stranded region can adopt two alternative conformations: a full-length duplex or a shorter duplex followed by a hairpin structure on the RNA3 acceptor template. The following mechanism of site-specific recombination was proposed (Figlerowicz, 2000): BMV replicase initiates nascent strand synthesis at the $3^{\prime}$ end of RNA1 (donor). Then the enzyme is paused at the $5^{\prime}$ end of a short homologous sequence, due to strong RNA/RNA interaction or hairpin formation on the acceptor template. Finally, the BMV replicase-nascent strand complex leaves the donor template, hybridizes to the homologous sequence located in acceptor RNA3 and resumes nascent strand synthesis. Heteroduplex-mediated recombination requires only local RNA1/ RNA3 hybridization and produces many different recombinants. Their junction sites are located close to each other and cluster in the left-hand portion of the local double-stranded structure. 
HS-RNA3 molecules (about 800-nt for parental and about 400-nt for recombinant RNA3). To determine whether recombinants were formed in infected cells, the RT-PCR products were analyzed by $1.5 \%$ agarose gel electrophoresis and their length was compared with the length of the cDNA amplified using primers $\mathrm{A}$ and $\mathrm{B}$ and in vitro transcribed Mag0-, Mag1-, Mag2- or HS-RNA3 as template. For selected recombinants, their presence in local lesions was additionally confirmed by Northern blot hybridization. Samples $(5 \mu \mathrm{l})$ of total RNA extracted from individual lesions were separated electrophoretically in $1 \%$ native agarose gel, stained with ethidium bromide (to test RNA stability), blotted onto a nylon membrane and probed with a ${ }^{32} \mathrm{P}$-labelled RNA probe. As probe we used in vitro transcribed 200-nt RNA complementary to the 3'UTR shared by all BMV genomic RNAs. Finally, RT-PCR products were cloned into the pGEM vector and sequenced to determine the location of recombinant junction sites. The full experiment involving each BMV mutant was repeated four times.

\section{RESULTS}

The effect of the DR7 mutation in the BMV polymerase gene on site-specific and heteroduplexmediated recombination

Earlier we found that a $C$ to $U$ substitution at position 1559 of BMV RNA2 (DR7 mutation, resulting in Leu-486 to Phe substitution in BMV polymerase) completely inhibits non-homologous recombination occurring within a local double-stranded region formed by BMV RNA1 and RNA3, without affecting the frequency of homology-driven recombination (Figlerowicz et al., 1997). We also showed that the DR7 mutation does not influence BMV infectivity and the ability of the virus to replicate and accumulate in plant cells. Taking advantage of the unique properties of the DR7 RNA2 mutant we used it to probe the mechanisms of site-specific and heteroduplex-mediated recombination. The experiment also involved three well-characterized BMV mutants: M1-BMV supporting both heteroduplex-mediated and site-specific recombination, M2-BMV supporting exclusively heteroduplex-mediated crossovers, and M0-BMV which does not support recombination (Figlerowicz, 2000). Their genomes are composed of wtRNA1, wtRNA2 and modified RNA3 (Mag1RNA3 in M1-BMV, Mag0-RNA3 in M0-BMV and Mag2-RNA3 in M2-BMV) (Table 1).

In all these mutants genomic molecules RNA1 and RNA3 are capable of forming similar local double-stranded structures (Fig. 2A). The $137 \mathrm{nt}$ wtRNA1/Mag1-RNA3 heteroduplex is accompanied by short homologous sequences $h$ and $h^{\prime}$ and con- sequently it can adopt two different conformations: a full-length duplex or a shorter duplex followed by a hairpin on the acceptor template (hairpin A) (Fig. 2B). In Mag0- and Mag2-RNA3, sequence $h$ or the complementary region c was removed from Mag1RNA3, respectively (Fig. 2A) (Figlerowicz, 2000). Accordingly, heteroduplexes formed by RNA1 and Mag0- or Mag2-RNA3 exist only in one conformation (Fig. 2B) (Figlerowicz \& Bibiłło, 2000).

To test how site-specific and heteroduplexmediated recombination is influenced by the DR7 mutation, a procedure described earlier was applied (Figlerowicz et al., 1997). C. quinoa plants (local lesion host for BMV) were inoculated with $\mathrm{M1}-$, M0and M2-BMV mutants as well as with their counterparts carrying the DR7 mutation: DR7M1-, DR7M0and DR7M2-BMV, respectively. The genomes of the three latter variants are composed of wtRNA1, DR7RNA2 and either Mag1-, Mag2- or Mag0-RNA3 (Table 1). After two weeks, the number of local lesions appearing on the inoculated leaves was counted. This confirmed our former observation that the DR7 mutation does not affect BMV infectivity (Table 1). Total RNA was extracted separately from each lesion, and the accumulation of BMV RNAs and the presence of RNA3 recombinants were analyzed by Northern blot hybridization (not shown). To determine the location of recombinant junction sites, the 3'-fragment of BMV RNA3 accumulating in each lesion (region where crossovers occur) was amplified by RT-PCR. The reaction products were cloned and sequenced (Fig. 3).

We observed that the exchange of wtRNA2 for the corresponding DR7 mutant did not change the recombination activity of the wtRNA1/Mag0RNA3 duplex - neither the M0- nor the DR7M0BMV mutant generated recombinants (Table 1). Total RNA extracted from approx. $70 \%$ of individual local lesions contained the parental virus (wtRNA1, wt or DR7-RNA2 and Mag0-RNA3). Interestingly, there was also a large fraction of so-called empty lesions (about 30\%) for which viral RNAs were not detected by RT-PCR or Northern blots. The DR7 mutation in RNA2 abolished the recombination activity of the wtRNA1/Mag2-RNA3 duplex (Table 1). M2-BMV supported exclusively heteroduplex-mediated recombination (Fig. 3C). Recombinants were identified in $60 \%$ of lesions. In the remaining $40 \%$ of lesions the parental virus was present. At the same time, recombinants did not form during infection with DR7M2-BMV (80\% of lesions contained the parental virus and in $20 \%$ no virus was detected). DR7-RNA2 also affected recombination between wtRNA1 and Mag1-RNA3. For M1-BMV, both site-specific and heteroduplex-mediated crossovers were observed, while for DR7M1-BMV only site-specific recombination occurred (12 and 6 recombinants of type A and $\mathrm{B}$, respectively) (Fig. 3A). In addition, recombination 
A.

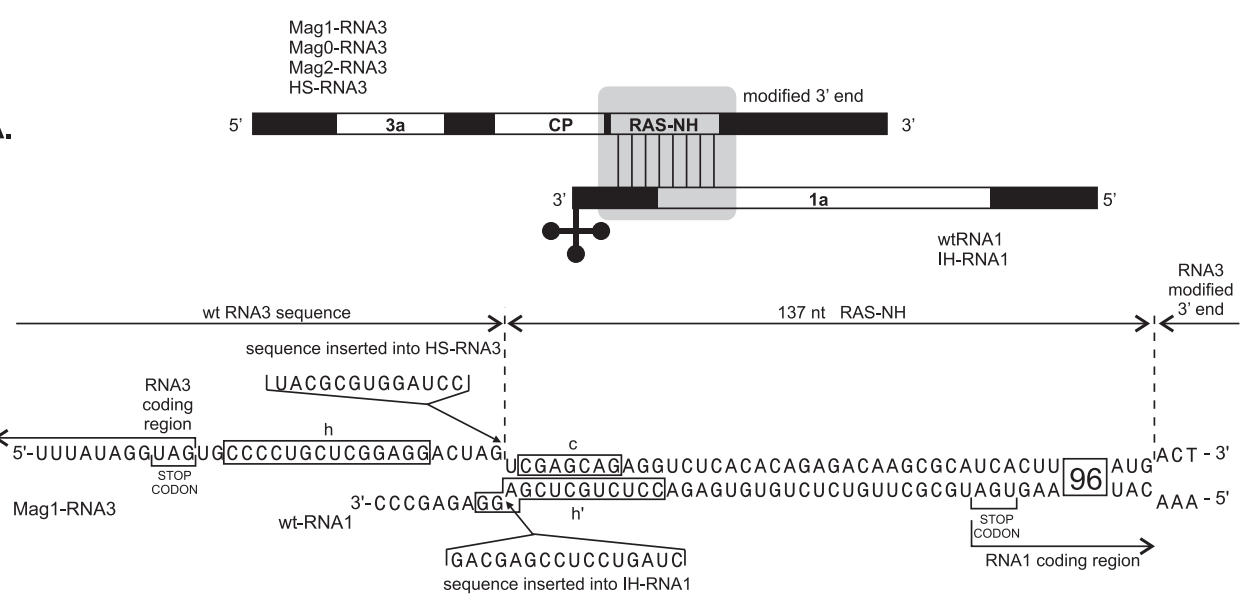

B.

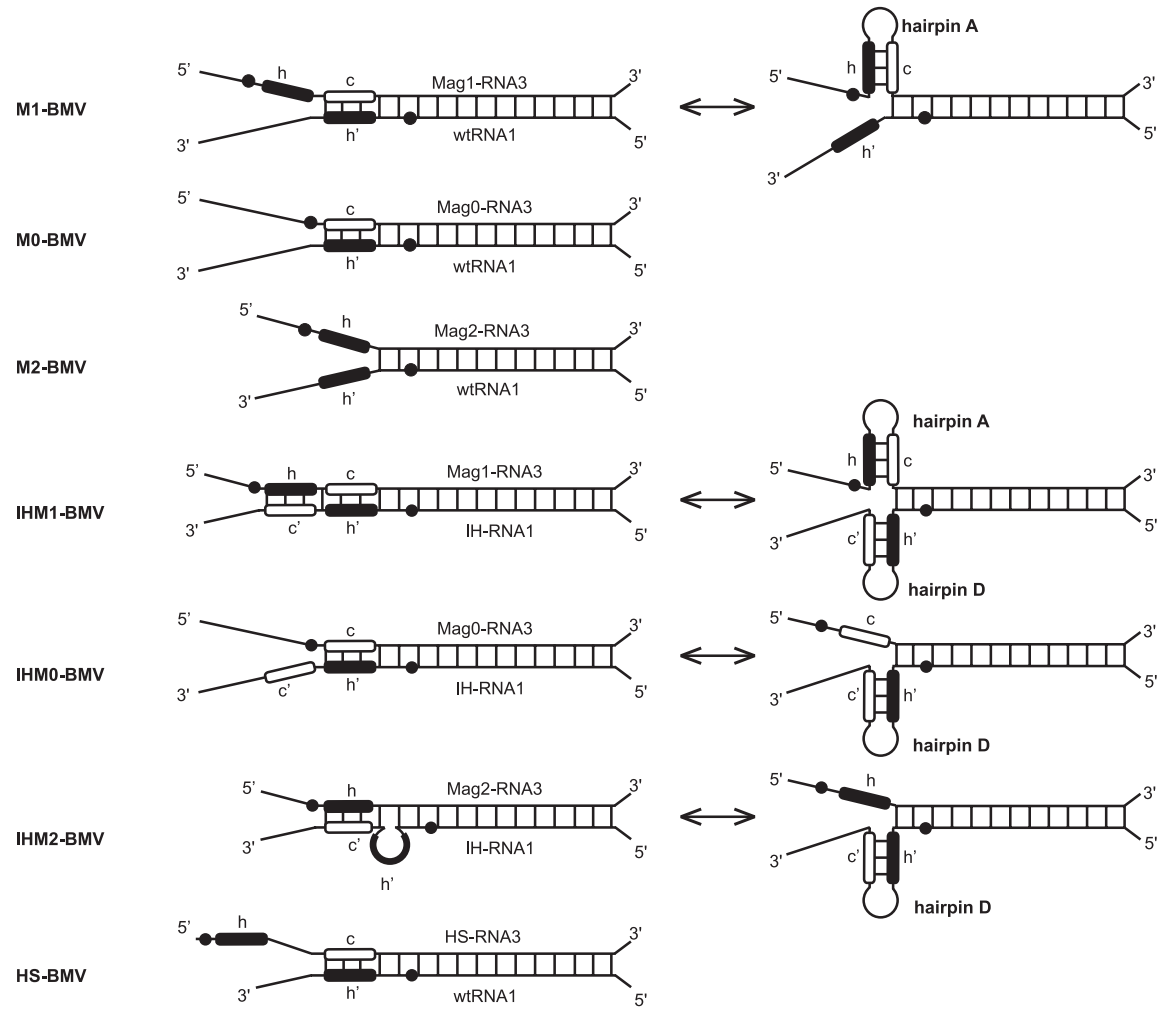

Figure 2. Schematic representation of the genomic molecules supporting non-homologous recombination in BMV mutants.

A. Recombinationally active RNA1 and RNA3 molecules. In all BMV mutants tested recombining RNA1 and RNA3 molecules were capable of forming a local double-stranded region (marked with a gray box and shown below). Initially, M1-BMV was constructed (Figlerowicz, 2000). Its genomic molecules wtRNA1 and Mag1-RNA3 formed a local double-stranded structure inducing both site-specific and heteroduplex-mediated crossovers (Figlerowicz, 2000). To construct Mag1-RNA3, a 137 nt sequence RAS-NH, complementary to RNA1 between positions 2856 and 2992, was inserted into RNA3 just behind the CP coding region. To obtain other mutants, specific modifications were introduced into Mag1-RNA3 or wtRNA1. Sequences $h$ and $c$ (boxed) were deleted from Mag1-RNA3 giving Mag0- and Mag2-RNA3, respectively (Figlerowicz, 2000). To obtain a new series of BMV mutants, HS-RNA3 and IH-RNA1 derivatives were constructed. HS-RNA3 was prepared by inserting a $13 \mathrm{nt}$ sequence into Mag1-RNA3, and IH-RNA1 by introducing a 16 nt sequence into wtRNA1 within its 3'UTR. The latter insert was complementary to RNA3 just before the local doublestranded region. B. Heteroduplexes that may be formed by wt or IH-RNA1 and modified Mag1-, Mag0-, Mag2- or HS-RNA3. Complementary fragments of RNA1 and RNA3 are shown schematically as in Fig. 1D. Black dots show the location of the stop codons in recombining molecules. The wtRNA1/Mag1-RNA3 duplex is accompanied by specifically located short homologous sequences $\mathrm{h}$ and $\mathrm{h}^{\prime}$ and thus it exists in two different conformations: a full-length duplex, and a shorter duplex followed by hairpin A forming on RNA3. Because of the changes introduced into Mag0- and Mag2RNA3, the heteroduplexes they can form with wtRNA1 adopt only one conformation. Due to the presence of sequences $\mathrm{c}^{\prime}$ and $\mathrm{h}^{\prime}$, a hairpin structure (hairpin D) can form on IH-RNA1. Therefore the latter may form a heteroduplex adopting two alternative conformations with all three: Mag1-, Mag0- and Mag2-RNA3 derivatives. In HS-RNA3, sequences h and $\mathrm{h}^{\prime}$ were separated to prevent hairpin A formation. Consequently, the wtRNA1/HS-RNA3 heteroduplex can only exist in one conformation. 
frequency was reduced for DR7M1-BMV, from 100 to $80 \%$. The remaining $20 \%$ of lesions contained the parental virus (Table 1).

\section{Preparation of new recombinationally active BMV mutants}

To increase our knowledge of the mechanism of site-specific and heteroduplex-mediated recombination a new series of BMV mutants was prepared. To this end, RNA1 and RNA3 derivatives named IH-RNA1 and HS-RNA3 were constructed (Fig. 2A). IH-RNA1 was made by inserting a 16 nt sequence (complementary to a portion of hairpin A) into the RNA1 3'-noncoding region. As a result, the putative IH-RNA1/Mag1-RNA3 duplex was extended (by 16 $\mathrm{nt})$ and sequences $\mathrm{h}$ and $\mathrm{c}$ involved in hairpin $\mathrm{A}$ formation were located within the double-stranded region. They base-paired with sequences named $h^{\prime}$ and $c^{\prime}$ that could potentially form a hairpin structure on IH-RNA1 (hairpin D) (Fig. 2B). To construct HS-RNA3, a 13 nt RNA fragment was inserted into Mag1-RNA3 between sequences $h$ and $c$, to separate short regions of homology ( $h$ and $h^{\prime}$ ) (Fig. 2A).
Using the previously prepared Mag0-, Mag1- and Mag2-RNA3 and the constructed IH-RNA1 and HSRNA3 derivatives, four new BMV mutants were obtained. In one, called HS-BMV, only RNA3 was modified, while in the three others named IHM0-, IHM1- and IHM2-BMV both RNA1 and RNA3 were changed (Table 1). In all mutants RNA1 and RNA3 were capable of forming similar local double-stranded structures, although in each the left-hand portion of the heteroduplex (the region where crossovers occur) was arranged in a different way (Fig. 2B).

\section{Infectivity and recombination activity of BMV mu- tants}

The infectivity and recombination activity of the mutants constructed were tested in C. quinoa plants. This revealed that HS- (with modified RNA3 only) and IHM0-BMV (possessing modified RNA1 and RNA3) were as infectious as the previously tested variants M0-, M1- and M2-BMV (in which only RNA3 was modified). The remaining two mutants IHM1- and IHM2-BMV, in which both genomic RNAs (RNA1 and RNA3) were modified, developed

Table 1. Infectivity and recombination activity of the BMV mutants

\begin{tabular}{|c|c|c|c|c|c|c|}
\hline BMV mutant & $\begin{array}{l}\text { Genomic } \\
\text { molecules }\end{array}$ & Infectivity * & $\begin{array}{l}\text { Number of } \\
\text { local lesions } \\
\text { analyzed }\end{array}$ & $\begin{array}{l}\text { Recombination } \\
\text { frequency } * * \\
(\%)\end{array}$ & $\begin{array}{l}\text { Lesions accumulating } \\
\text { parental RNA3 } \\
(\%)\end{array}$ & $\begin{array}{l}\text { Empty lesions } \\
(\%)\end{array}$ \\
\hline M1-BMV & $\begin{array}{l}\text { wtRNA1 } \\
\text { wtRNA2 } \\
\text { Mag1-RNA3 }\end{array}$ & $18( \pm 4)$ & 40 & 100 & 0 & 0 \\
\hline M0-BMV & $\begin{array}{l}\text { wtRNA1 } \\
\text { wtRNA2 } \\
\text { Mag0-RNA3 }\end{array}$ & $20( \pm 3)$ & 40 & 0 & 70 & 30 \\
\hline M2-BMV & $\begin{array}{l}\text { wtRNA1 } \\
\text { wtRNA2 } \\
\text { Mag2-RNA3 }\end{array}$ & $18( \pm 3)$ & 40 & 60 & 40 & 0 \\
\hline DR7M1-BMV & $\begin{array}{l}\text { wtRNA1 } \\
\text { DR7-RNA2 } \\
\text { Mag1-RNA3 }\end{array}$ & $17( \pm 4)$ & 40 & 80 & 20 & 0 \\
\hline DR7M0-BMV & $\begin{array}{l}\text { wtRNA1 } \\
\text { DR7-RNA2 } \\
\text { Mag0-RNA3 }\end{array}$ & $20( \pm 5)$ & 40 & 0 & 70 & 30 \\
\hline DR7M2-BMV & $\begin{array}{l}\text { wtRNA1 } \\
\text { DR7-RNA2 } \\
\text { Mag2-RNA3 }\end{array}$ & $19( \pm 4)$ & 40 & 0 & 80 & 20 \\
\hline IHM1-BMV & $\begin{array}{l}\text { IH-RNA1 } \\
\text { wtRNA2 } \\
\text { Mag1-RNA3 }\end{array}$ & $10( \pm 3)$ & 40 & 80 & 15 & 5 \\
\hline IHM0-BMV & $\begin{array}{l}\text { IH-RNA1 } \\
\text { wtRNA2 } \\
\text { Mag0-RNA3 }\end{array}$ & $18( \pm 3)$ & 40 & 15 & 55 & 30 \\
\hline IHM2-BMV & $\begin{array}{l}\text { IH-RNA1 } \\
\text { wtRNA2 } \\
\text { Mag2-RNA3 }\end{array}$ & $3( \pm 2)$ & 40 & 60 & 40 & 0 \\
\hline HS-BMV & $\begin{array}{l}\text { wtRNA1 } \\
\text { wtRNA2 } \\
\text { HS-RNA3 }\end{array}$ & $16( \pm 3)$ & 40 & 95 & 5 & 0 \\
\hline
\end{tabular}

${ }^{*}$ Infectivity is defined as the average number of lesions per leaf; ${ }^{* *}$ Recombination frequency is defined as the ratio between the number of lesions that developed recombinants to the total number of analyzed lesions. 
a much lower number of lesions (Table 1). Individual local lesions were then excised and total RNA was extracted separately from each lesion and subjected to a three-step analysis (representative results obtained for the newly generated IHM0- IHM1- and IHM2-BMV mutants are shown in Fig. 4). The recombination frequency was counted for HS-, IHM0-, IHM1-, and IHM2-BMV and compared with the analogous data obtained earlier for M0-, M1- and M2-
BMV (Figlerowicz, 2000) (Table 1). We found that HS-BMV generated recombinants with the same high frequency (95\%) as M1-BMV, although in the former the homologous sequences $h$ and $h^{\prime}$ were separated. Interestingly, with the exchange of wtRNA1 (present in M1-, M0- and M2-BMV) for IH-RNA1, the recombination frequency was reduced for IHM1-BMV (from 100 to $80 \%$ ), increased for IHM0-BMV (from 0 to $15 \%)$ and unchanged for IHM2-BMV (60\%).

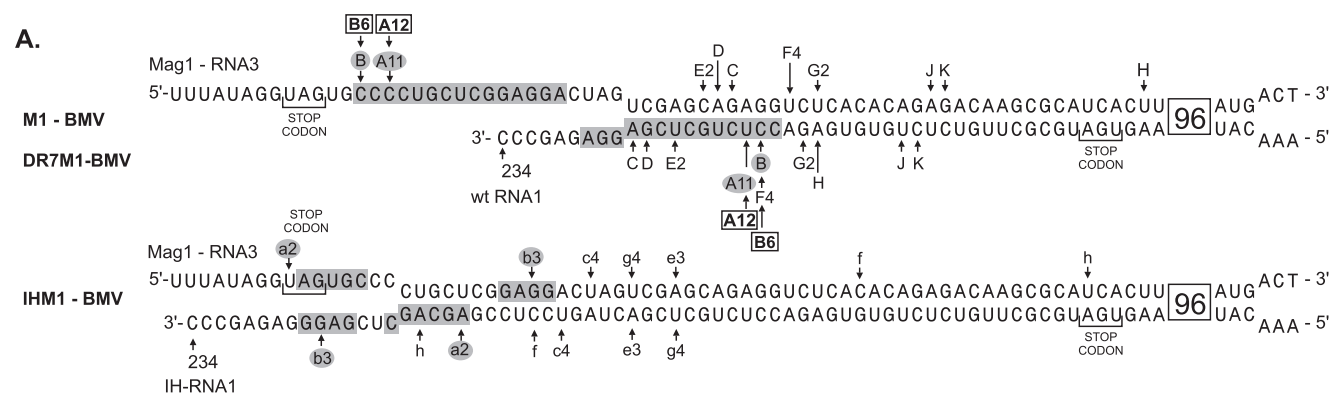

B.

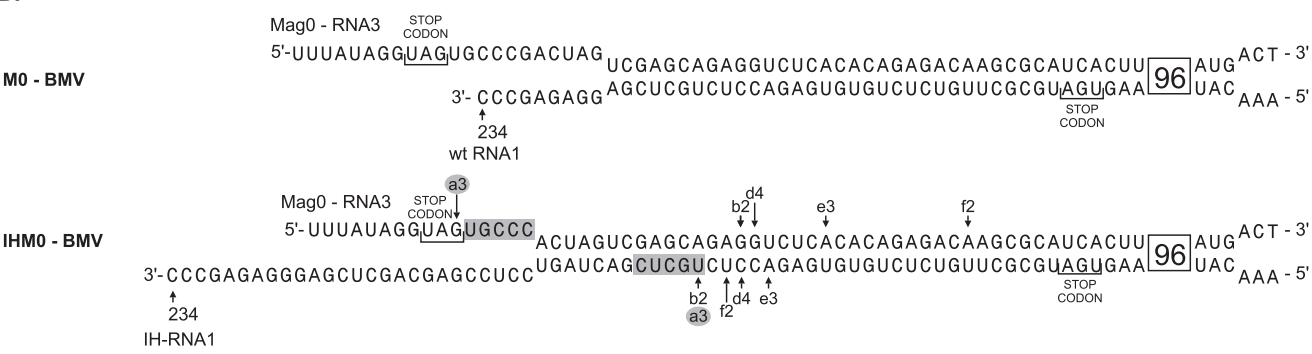

c.

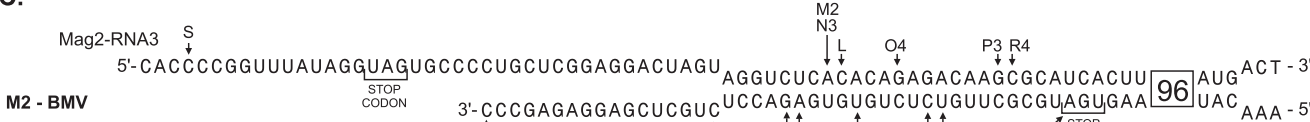

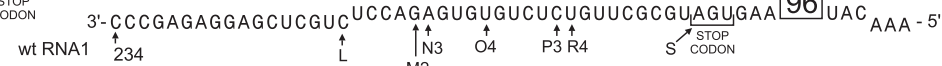

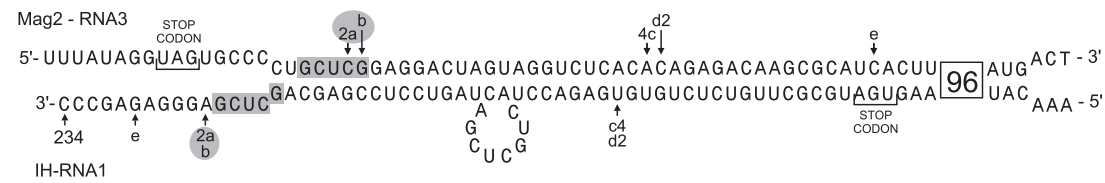

D.

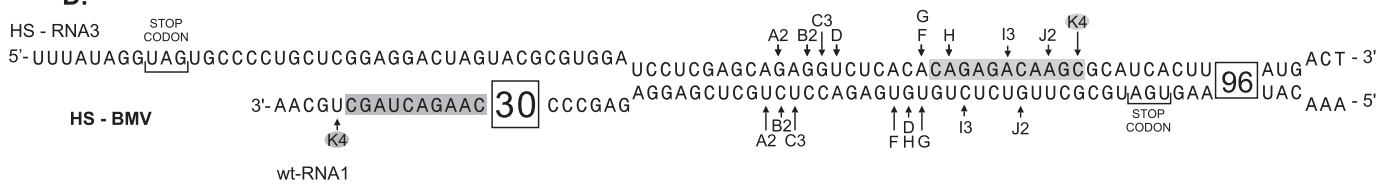

Figure 3. Distribution of non-homologous crossovers occurring in vivo in C. quinoa plants infected with the BMV mutants.

Each recombinant was isolated from a separate local lesion. In every case recombining RNA1 and RNA3 molecules were capable of forming 130-150 nt local double-stranded regions. However, in the picture only the left-hand portion of the duplex is presented - the region where recombinant junction sites are located. In each duplex the upper sequence represents the (+) strand of the RNA3 derivative (the region where RAS is located), while the lower sequence represents the corresponding (complementary) fragment of the (+) RNA1 strand. The recombinant junction sites are marked with arrows (pointing to the last nucleotide coming from RNA1 and the first nucleotide from the RNA3 derivative), and either with capital letters (for recombinants generated with wtRNA1 and wtRNA2), boxed capital letters (for recombinants generated with wtRNA1 and DR7 RNA2 mutant) or lower case letters (for recombinants generated with IH-RNA1 and wtRNA2). Numbers accompanying the letters indicate how often the given recombinant was identified. Recombinants whose formation was most likely mediated by short homologous sequences as well as homologous regions are shadowed. Recombinants generated with: M1-, DR7M1- and IHM1-BMV (A); M0- and IHM0-BMV (B); M2- and IHM2-BMV (C); HS-BMV (D). 
A.
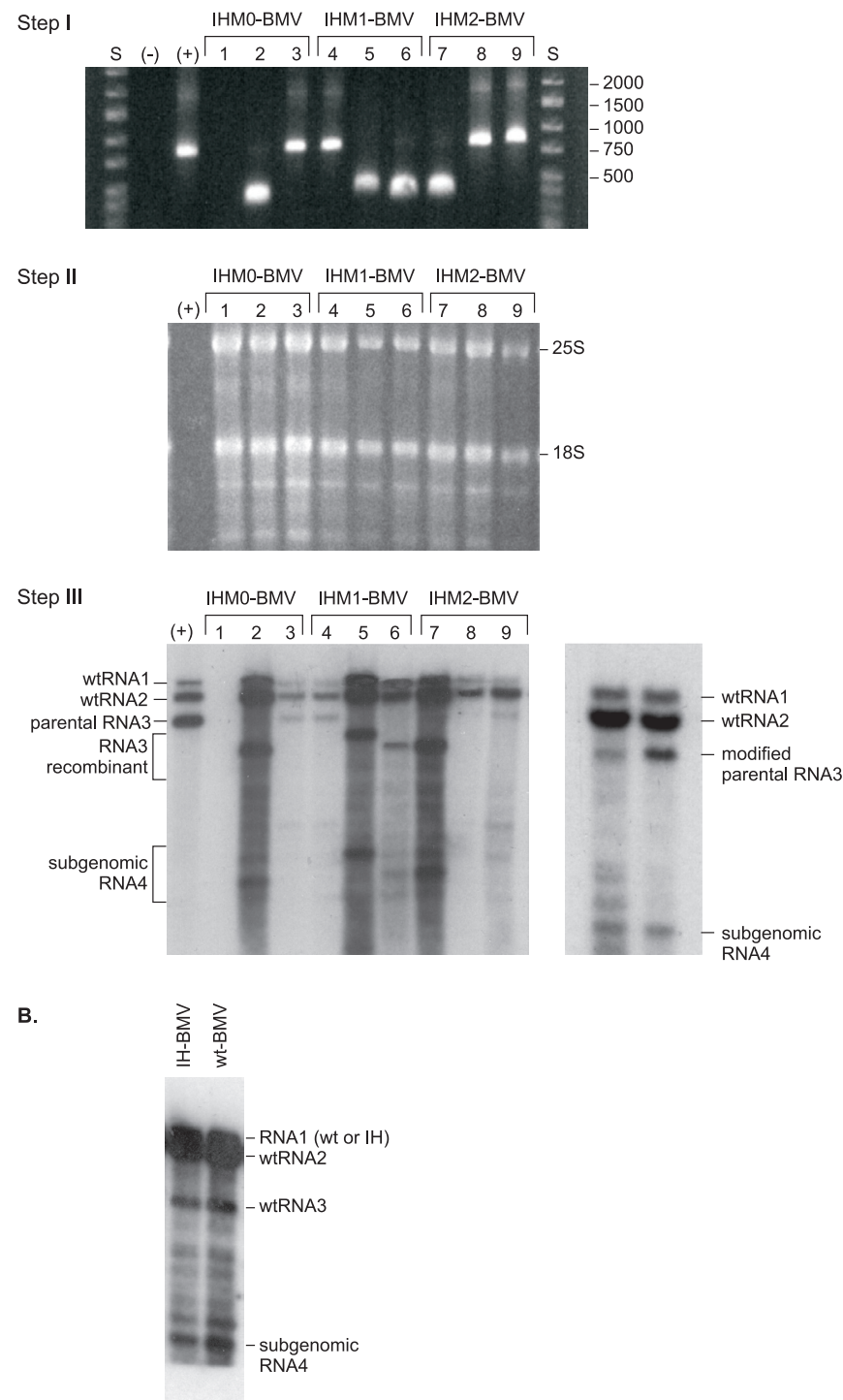

Figure 4. Three-step analysis of BMV progeny RNAs accumulating in C. quinoa plants infected with IHM0-, IHM1and IHM2-BMV.

Representative results obtained for IHM0-, IHM1- and IHM2-BMV mutants are shown. Two weeks after inoculation, individual local lesions developed on C. quinoa leaves were excised, and total RNA was extracted separately from each lesion and dissolved in $50 \mu \mathrm{l}$ of TE buffer. A. Step I - RT-PCR analysis of BMV RNA3. Samples of $3.5 \mu$ of total RNA were subjected to RT-PCR involving primers A and B (Fig. 1) specific for the amplification of the RNA3 3'-fragment (region where recombination crossovers occur). In addition, two RT-PCR reactions in which the total RNA was replaced either with a 100-times diluted mixture of BMV RNA transcripts (used for inoculation) or with water, were carried out as a positive (lane + ) and negative (lane -) control, respectively. RT-PCR products were separated in a 1.5\% agarose gel and visualized by ethidium bromide staining. Their lengths were estimated by comparison with a DNA molecular mass marker (lanes S). The presence of longer products (about $800 \mathrm{nt}$ - identical to lane +) suggested that parental RNA3 accumulated (lanes: 3, 4, 8 and 9) while the formation of shorter products (about 400-500 nt) was a sign of RNA3 recombinant formation (lanes: 2, 5, 6 and 7). The lack of an RT-PCR product indicated that BMV RNA3 was absent in the lesion analyzed or accumulated below RT-PCR detection level (lane 1). Step II - stability of the total RNA extracted. Samples $(5 \mu \mathrm{l})$ of total RNA were subjected to electrophoresis in a native $1 \%$ agarose gel. After ethidium bromide staining the most abundant RNAs were visualized. Positions of the clearly visible bands corresponding to $18 \mathrm{~S}$ and $25 S$ ribosomal RNA are marked. Identical results were obtained for all BMV mutants tested, suggesting that the RNA extracted was stable in every sample analyzed. Step III - Northern blot analysis of BMV RNA accumulation. Left panel - representative data obtained for the BMV mutants tested. Right panel - typical BMV RNA accumulation in lesions which did not contain recombinants. Total RNA separated in a native 1\% agarose gel and stained with ethidium bromide (to test RNA stability) was blotted onto a nylon membrane and probed with a ${ }^{32}$ P-labelled RNA (200 nt probe complementary to the $3^{\prime}$ UTR shared by all BMV genomic RNAs). A mixture of BMV RNA transcripts was applied as a molecular mass marker (lane + ). The results obtained were consistent with those of RT-PCR analysis. RNA3 recombinants were present in four lesions (lanes: 2, 5, 6 and 7), parental RNA3 molecules were detected in four lesions (lanes: 3, 4, 8 and 9), while in one lesion neither recombinant nor parental RNA could be found (lane 1). BMV RNAs accumulated to high levels only in those lesions in which an RNA3 recombinant was formed. Interestingly, in the lesions accumulat- 
Eighty percent of the lesions formed on leaves inoculated with IHM1-BMV contained recombinants, $15 \%$ accumulated parental Mag1-RNA3 and in 5\% the virus was not detected (empty lesions). In all lesions developed by M2-, HS- and IHM2-BMV, parental or recombinant RNA3 was present. At the same time, RT-PCR analysis suggested that RNA3 was absent or accumulated below RT-PCR detection level in a large fraction (about $30 \%$ ) of the lesions excised from M0- and IHM0-BMV infected plants. M0-BMV did not produce recombinants, whereas IHM0-BMV, in addition to empty lesions and lesions accumulating the parental virus (55\%), also developed lesions containing recombinants $(15 \%)$. To exclude the possibility that in some samples RNA was occasionally degraded, $5 \mu$ l of the total RNA extracted separately from each lesion was analyzed by agarose gel electrophoresis. After ethidium bromide staining, most abundant cellular RNAs were visualized (Fig. 4, Step II). The results obtained demonstrate that every sample comprised a similar amount of RNA, suggesting that the extracted molecules were not degraded during isolation. Moreover, our data prove that the total RNA was always isolated from a similar portion of plant tissue.

Finally, the accumulation of viral RNA was determined by Northern blot hybridization (Fig. 4, Step III). This showed a prominent accumulation of BMV RNAs only in those lesions where an RNA3 recombinant was formed (Fig. 4, e.g. lanes: 2, 5, 6 and 7 - about $95 \%$ of lesions contained only one recombinant, sometimes, however, two recombinants were generated). In other lesions viral RNAs accumulated either to a very low level (Fig. 4, e.g. lanes: 3, 4, 8 and 9 - lesions containing the parental virus) or below detection level (e.g. lane 1). Interestingly, in the lesions accumulating the parental virus (lesions in which recombinants were not generated), RNA1 and RNA3, i.e. the molecules carrying long complementary sequences occurred in a visibly lower concentration than RNA2 (Fig. 4, Step III).

To determine whether BMV infection is affected by a modification introduced into IH-RNA1, C. quinoa plants were inoculated either with wt BMV or an IH-BMV mutant. The genome of the latter was composed of IH-RNA1, wt RNA2 and wt RNA3. After two weeks, the number of developed lesions was counted, total RNA was extracted from 20 indi- vidual lesions (for each BMV variant) and the accumulation of viral RNAs was analyzed by Northern blot hybridization as described above. Additionally, the extracted total RNA was subjected to RT-PCR involving primers which enabled a selective amplification of the RNA1 3'-fragment (the region where the modification was introduced). The products obtained were cloned and sequenced. We found that wt BMV and IH-BMV accumulate in plant cells to similar levels and IH-RNA1 maintains its modification during the entire infection period (Fig. 4B).

\section{Location of recombinant junction sites}

To establish the location of recombinant junction sites, the RT-PCR products of selective RNA3 3 '-end amplification were cloned and sequenced (Fig. 3). As earlier, we observed that recombination crossovers occur within the left-hand portion of RNA1/RNA3 heteroduplex (or in close vicinity) (Figlerowicz, 2000). A detail analysis of the recombinants identified (also those generated during M0-, M1- and M2-BMV infection) showed that they can be divided into two groups. The first group is composed of recombinants whose junction sites are located within short homologous sequences (homology-dependent recombinants). One of the sequences is always located within the local double-stranded region, while the second sequence lies before a heteroduplex. The most typical examples of homology-dependent recombinants are the products of site-specific recombination (recombinants $\mathrm{A}$ and $\mathrm{B}$ ) generated with M1-BMV (Fig. 3A). However, such recombinants were also formed during infection involving other mutants, e.g. recombinants a and b obtained for IHM1- and IHM2-BMV or recombinants a and $\mathrm{K}$ produced in plants infected with IHM0- and HS-BMV, respectively (Fig. 3).

The second group comprises recombinants for which there is no homology within the crossover region (homology-independent recombinants). Their junction sites are usually located close to each other within the RNA1/RNA3 heteroduplex structure. Most frequently they are separated by $2-3 \mathrm{nt}$. The additional regularity noted for both homologydependent and homology-independent recombinants was that their junction sites were located exclusively in the non-coding fragments of RNA1 and RNA3 -

\section{Figure 4. Continued}

ing parental RNA3, BMV genomic molecules carrying complementary sequences (i.e. RNA1 and RNA3) were always in a much lower concentration than RNA2 (this is better shown in the right panel). The three-step analysis of BMV RNA accumulation was carried out for all mutants tested. B. Northern blot analysis of BMV RNA accumulation in lesions induced by IH-BMV and wt-BMV infection. To determine whether BMV infection is affected by a modification introduced into IH-RNA1, the accumulation of viral RNAs in C. quinoa plants inoculated either with wt BMV or IH-BMV mutant was compared by Northern blot hybridization, as described above. wt BMV and IH-BMV accumulated in plant cells to similar levels. 
in the portion of the heteroduplex which is placed between the stop codons present in the recombining molecules. The only exception was recombinant S (generated with M2-BMV) (Figlerowicz, 2000). One of its junction sites was located in the RNA3 coding region and therefore it encoded a mutated but functional coat protein. Nevertheless this recombinant $S$ accumulated in infected plants to a similar level as other recombinants.

\section{DISCUSSION}

Two types of non-homologous recombination in BMV

More than ten years ago Nagy and Bujarski (1993) demonstrated that non-homologous crossovers occur within the local complementary regions present in BMV RNA1 and RNA3. They postulated that RNA1/RNA3 base-pairing causes the BMV replicase to pause and accidentally induces template switching from the donor RNA1 to the acceptor RNA3 molecule. Our recent studies revealed that there may exist two different types of non-homologous recombination in BMV: site-specific and heteroduplex-mediated (Figlerowicz, 2000). Comparison of the recombination activities of M1-, M2-BMV and their counterparts carrying the DR7 mutation provided strong evidence supporting the above assumption. The diverse effects of the modified BMV polymerase on site-specific and heteroduplex-mediated crossovers indicate that different mechanisms operate in both types of recombination. Based on our earlier observations, on the influence of DR7-2a protein on homologous and non-homologous crossovers, showing that the former are unaffected and the latter inhibited (Figlerowicz et al., 1997), one can draw the following conclusions: (i) the lack of any effect of the DR7 mutation on the frequency of sitespecific recombination suggests that short homologous sequences ( $h$ and $h^{\prime}$ ) play a key role in this process, and (ii) the selective inhibition of heteroduplex-mediated recombination indicates that it does not depend on homology.

\section{The mechanism of site-specific recombination}

Considering the effect of the DR7 mutation on site-specific and heteroduplex-mediated recombination one could speculate that the former should be classified as homologous. This is, however, contradicted by the fact that site-specific recombinants A and B do not form for M2- and HS-BMV although sequences $h$ and $h^{\prime}$ are present in recombining molecules (Fig. 3C and D). This means that relatively short regions of homology are not sufficient to mediate frequent crossovers. The positioning of ho- mologous sequences also seems to be important. In general, our former (Figlerowicz, 2000) and current observations suggest that there are two basic requirements for site-specific recombination: local hybridization between recombining molecules and the presence of two short homologous sequences specifically placed in the donor and acceptor templates. One sequence must be located within a local double-stranded region (on donor RNA) and the second before the RNA1/RNA3 heteroduplex (on acceptor RNA). The frequency of site-specific crossovers is also affected by the distance between homologous sequences and by their length. That is why M1-BMV possessing the $14 \mathrm{nt}$ sequences $\mathrm{h}$ and $\mathrm{h}^{\prime}$ placed close to each other (h just before and $h^{\prime}$ in the very beginning of the RNA1/Mag1-RNA3 heteroduplex) supports site-specific recombination very efficiently while it is inhibited or less frequent during infections involving other BMV mutants in which the recombination hot-spot was modified.

The data presented show that the wtRNA1/ Mag1-RNA3 heteroduplex offers the best conditions for site-specific template switching by BMV replicase. They also suggest that recombinants $\mathrm{A}$ and $\mathrm{B}$ are formed during (-)RNA strand synthesis. BMV replicase starts nascent strand synthesis on (+)RNA1, it pauses within the local double-stranded region and is then transferred to sequence $h$ located very near the RNA1/Mag1-RNA3 heteroduplex. The alternative assumption that crossovers occur during (+) strand synthesis would mean that the polymerase is stopped just before the heteroduplex and then switches to sequence $h$, located within the doublestranded region. By analogy, the formation of recombinant $\mathrm{K}$ during HS-BMV infection, recombinant $b$ during IHM1-BMV infection or recombinants a and b during IHM2-BMV infection indicates that nothing hinders site-specific crossovers from occurring also during (+)RNA strand synthesis. Interestingly, in spite of the similar spatial arrangement of recombination hot-spots in RNA1/Mag1-RNA3 and (-)IHRNA1/(-)Mag0-RNA3 heteroduplexes, the latter did not support site-specific crossovers during IHM0BMV infection. This might suggest that in addition to RNA primary and secondary structure there are some other still unrecognized factors affecting sitespecific recombination.

\section{Mechanism of heteroduplex-mediated recombination}

Unfortunately, at present one cannot unambiguously define the mechanism of heteroduplexmediated recombination. At first glance, it seems quite obvious that a stable double-stranded RNA structure can accidentally enforce template switching by BMV replicase, as was proposed by Nagy and Bujarski (1993). However, the data collected in the recent years have raised several questions that 
cannot be answered based on this model. It does not explain why recombinant junction sites are located almost exclusively within the left-hand portion of RNA1/RNA3 heteroduplexes. It was demonstrated that the BMV replicase can easily unwind even a 200-500 nt duplex, since it is equipped with RNA helicase (Ahlquist et al., 1984). Consequently, one would expect roughly random template switching by the viral polymerase along the local RNA1/RNA3 heteroduplex. Moreover, it is difficult to find any factor that would facilitate the transfer of the BMV replicase/nascent strand complex between complementary templates (Figlerowicz, 2000). In other types of recombination this role is played by short homologous sequences (e.g. in site-specific recombination) or by promoter-like structures (Nagy \& Simon, 1997). The next questions are why, before recombinant formation, wtRNA1 and RNA3 replicate to a visibly lower level than RNA2 (Fig. 4, Step III) if the only modified molecule is RNA3, which is dispensable for genomic RNA synthesis (Ahlquist, 1992), or why recombinant junction sites are placed between the stop codons that are present in the RNA1 and RNA3 molecules.

Nagy and Bujarski's model also presupposes that during BMV genome replication, efficient RNA1/RNA3 hybridization occurs. This seems to be very unlikely if one considers the recent model of BMV RNA replication (Schwartz et al., 2002): it assumes that viral genomic molecules are synthesized in 50-70 $\mathrm{nm}$ spherules budding into the endoplasmic reticulum membranes. Spherule formation is induced by the BMV 1a protein anchoring to the cytoplasmic face of the membranes. Usually, only one RNA template and one nascent strand are located inside the spherule forming a ribonuclease-resistant shell, in which RNA replication occurs. Initially the genomic $(+)$ RNA strand serves as a template. It is copied and then released through a narrow neck connecting the spherule with the cytoplasm. The (-)RNA strand remains inside the spherule and is used as a template for $(+)$ strand synthesis.

All the above observations suggest that some additional, still unknown factors affect heteroduplex-mediated recombination in BMV. Considering three facts - (i) recombining RNA1 and RNA3 molecules are capable of forming a long, double-stranded structure; (ii) RNA1 and RNA3 molecules carrying complementary sequences accumulate to a visibly lower level than RNA2; and (iii) BMV mutants which infrequently produce recombinants and consequently cannot remove complementary sequences from RNA1 and RNA3 are often eliminated from infected cells (formation of empty lesions is observed) - one can speculate that sequence-specific doublestranded RNA degradation, referred to as RNA silencing, is one of the factors influencing template switching within the RNA1/RNA3 heteroduplex.
Since the latter is also indispensable during site-specific crossovers, it cannot be excluded that they are affected by RNAi, too. However, further studies are required to prove that there is some interplay between non-homologous recombination in BMV and RNA silencing.

\section{Acknowledgments}

The authors would like to thank Anne-Lise Haenni, Zofia Szweykowska-Kulińska, Witold Filipowicz and Józef J. Bujarski for their advice and comments on the manuscript. We also thank Zbigniew Michalski for his assistance in the preparation of figures.

This research was supported by the Polish Government through grant (6 P04A 038 19) from the State Committee for Scientific Research (KBN).

\section{REFERENCES}

Ahlquist P (1992) Bromovirus RNA replication and transcription. Curr Opin Genet Dev 2: 71-76.

Ahlquist P, Bujarski JJ, Kaesberg P, Hall TC (1984) Localization of the replicase recognition site within brome mosaic virus RNA by hybrid-arrested RNA synthesis. Plant Mol Biol 3: 37-44.

Alejska M, Kurzyńska-Kokorniak A, Broda M, Kierzek R, Figlerowicz M (2001) How RNA viruses exchange their genetic material. Acta Biochim Polon 48: 391-407.

Bujarski JJ, Kaesberg P (1986) Genetic recombination in a multipartite plant virus. Nature 321: 528-531.

Eigen M (1996) On the nature of virus quasispecies. Trends Microbiol 4: 216-217.

Figlerowicz M (2000) Role of RNA structure in non-homologous recombination between genomic molecules of brome mosaic virus. Nucleic Acids Res 28: 1714-1723.

Figlerowicz M, Bujarski JJ (1998) RNA recombination in brome mosaic virus, a model plus stranded RNA virus. Acta Biochim Polon 45: 1-23.

Figlerowicz M, Bibiłło A (2000) RNA motifs mediating in vivo site-specific nonhomologous recombination in $(+)$ RNA virus enforce in vitro nonhomologous crossovers with HIV-1 reverse transcriptase. RNA 6: 339-351.

Figlerowicz M, Nagy PD, Bujarski JJ (1997) A mutation in the putative RNA polymerase gene inhibits nonhomologous, but not homologous, genetic recombination in RNA virus. Proc Natl Acad Sci USA 94: 2073-2078.

Figlerowicz M, Alejska M, Kurzyńska-Kokorniak A, Figlerowicz M (2003) Genetic variability - the key problem in prevention and therapy of RNA-based virus infections. Med Res Rev 23: 488-518.

Khatchikian D, Orlich M, Rott R (1989) Increased viral pathogenicity after insertion of a $28 \mathrm{~S}$ ribosomal RNA sequence into the hemagglutinin gene of an influenza virus. Nature 340: 156-157.

Kroner PA, Young BM, Ahlquist P (1990) Analysis of the role of brome mosaic virus 1a-protein domains in RNA replication, using linker insertion mutagenesis. J Virol 64: 6110-6120.

Lai MMC (1992) RNA recombination in animal and plant viruses. Microbiol Rev 56: 61-79.

Meyers G, Tautz N, Dubovi EJ, Thiel H-J (1991) Viral cytopathogenicity correlated with integration of ubiquitincoding sequences. Virology 180: 602-616. 
Nagy PD, Bujarski JJ (1992) Genetic recombination in brome mosaic virus: effect of sequence and replication of RNA on accumulation of recombinants. I Virol 66: 6824-6828.

Nagy PD, Bujarski JJ (1993) Targeting the site of RNARNA recombination in brome mosaic virus with antisense sequences. Proc Natl Acad Sci USA 90: 6390-6394.

Nagy PD, Simon AE (1997) New insights into the mechanism of RNA recombination. Virology 235: 1-9.

Pathak VK, Hu W-S (1997) „Might as well Jump!” Template switching by retroviral reverse transcriptase, de- fective genome formation and recombination. Semin Virol 8: 141-150.

Schwartz M, Chen J, Janda M, Sullivan M, den Boon J, Ahlquist P (2002) A positive-stranded RNA virus replication complex parallels form and function of retrovirus capsids. Mol Cell 9: 505-514.

Traynor P, Young BM, Ahlquist P (1991) Deletion analysis of brome mosaic virus $2 \mathrm{a}$ protein: effects on RNA replication and systemic spread. J Virol 65: 2807-2815. 